\title{
High Sensitive and Wide Detecting Range MOS Tunneling Temperature Sensors for On-Chip Temperature Detection
}

\author{
Yen-Hao Shih, Shian-Ru Lin, Tsung-Miau Wang, and Jenn-Gwo Hwu, Senior Member, IEEE
}

\begin{abstract}
This paper examined the feasibility of applying a highly sensitive metal-oxide-semiconductor (MOS) tunneling temperature sensor, which was compatible with current CMOS technology. As the sensor was biased positively at a constant voltage, the gate current increased more than 500 times when the sensor was heated from $20^{\circ} \mathrm{C}$ to $110{ }^{\circ} \mathrm{C}$. However, when the sensor was biased at a constant-current situation, its gate voltage magnitude changed significantly with substrate temperature, with a sensitivity exceeding $-2 \mathrm{~V} /{ }^{\circ} \mathrm{C}$. The improvement of temperature sensitivity in this paper is one thousand times over the sensitivity of a conventional p-n junction, i.e., namely, about $-2 \mathrm{mV} /{ }^{\circ} \mathrm{C}$. Regarding a temperature sensor array, this paper proposes a method using gate current gain, rather than absolute gate current, to eliminate the gate current discrepancy among sensors. For constant current operation, a sensitivity exceeding 10 $\mathrm{V} /{ }^{\circ} \mathrm{C}$ can be obtained if the current level is suitable. Finally, this paper demonstrates a real temperature distribution for on-chip detection. With such a high temperature-sensitive sensor, accurate temperature detection can be incorporated into common CMOS circuits.
\end{abstract}

Index Terms-Metal-oxide-semiconductor (MOS) device, temperature sensor, ultrathin oxide.

\section{INTRODUCTION}

D ECREASING feature sizes and increasing packaging densities have increased power densities in integrated circuits (ICs), and decreased the reliability of these systems. Early detection of possible overheating problems can prevent system malfunctioning due to irreversible damage caused by the increased temperatures. Thermal monitoring is extremely important in the case of safety-critical IC. Circuits used for the control of railway systems, space, aeronautics, and automotive critical functions require concurrent error detection for both tran-

Manuscript received October 15, 2003; revised April 5, 2004. This work was supported by the National Science Council, Taiwan, R.O.C., under Contract NSC 92-2120-E-002-005. The review of this paper was arranged by Editor K. Najafi.

Y.-H. Shih is with Emerging Central Lab, Macronix International Company, Ltd., Hsinchu, Taiwan, R.O.C.

S.-R. Lin is with Realtek Semiconductor Corporation, Hsinchu, Taiwan, R.O.C.

T.-M. Wang is with the Department of Electrical Engineering/Graduate Institute of Electronics Engineering, National Taiwan University, Taipei, Taiwan, R.O.C.

J.-G. Hwu is with the Department of Electrical Engineering/Graduate Institute of Electronics Engineering, National Taiwan University, Taipei, Taiwan, R.O.C. He is also with the Department of Electronics Engineering/Department of Electrical Engineering, National United University, Miaoli, Taiwan, R.O.C. (e-mail: hwu@cc.ee.ntu.edu.tw).

Digital Object Identifier 10.1109/TED.2004.833571 sient and permanent faults; a goal that can be achieved only by making the circuits self-checking.

IC designers usually do not consider the thermal system in detail when designing an electrical circuit. In numerous cases, the effects of the thermal system are negligible. However, as technology downscaling causes greater power and package densities, thermal effects become significant in increasing numbers of designs. Facing thermal problems is a new challenge for electronic engineers. Until recently, electronic engineers generally left these problems to mechanical engineers, who applied external cooling to the warming packages or sometimes directly to the chips. It was reported that even a $1{ }^{\circ} \mathrm{C}$ increase could increase IC failure rate by $2 \%-4 \%$ [1]. Without an appropriate thermal solution [2], chips are easily burnt out in seconds.

An ideal thermal solution includes: 1) chip temperature detection, and 2) methods for chip cooling. Using an on-chip temperature sensor is known to further enhance precision and accuracy. For example, a transistor [3]-[6] or a p-n junction diode [7]-[9] is preferred, owing to its easy fabrication and well-known physics. However, the conventional temperature sensors generally have a lower temperature sensitivity. For example, the temperature sensitivity in a p-n junction is around $-2 \mathrm{mV} /{ }^{\circ} \mathrm{C}$; that is, when the temperature increases by $1{ }^{\circ} \mathrm{C}$, the extra junction voltage must reduce by about $2 \mathrm{mV}$ to maintain the same current magnitude. Circuit designers usually use the emitter-to-base voltage of a biploar junction transistor to detect temperature. Additionally, the temperature also could be detected by measuring the threshold voltage $\left(V_{T}\right)$ of an MOSFET. The temperature sensitivity of $V_{T}$ is within the range of -0.5 to $-4 \mathrm{mV} /{ }^{\circ} \mathrm{C}$. Our recent paper demonstrated that a simple metal-oxide-semiconductor (MOS) capacitor with an oxide thickness $t_{\mathrm{ox}}$ of around $2 \mathrm{~nm}$ could be suitable for adoption as an on-chip temperature sensor [10]. Moreover, the on-chip temperature sensors can be integrated into the circuit with an improved sensitivity of $-2 \mathrm{~V} /{ }^{\circ} \mathrm{C}$. The improvement of temperature sensitivity was exceeded three orders of magnitude compared with that of a p-n junction. This paper gives the detailed considerations involved in biasing the MOS temperature sensors, including constant voltage and constant current operations, and also gives fabrication notices. The current gain method and multipoints mapping technologies are introduced particularly show the applicability of using MOS diodes as temperature sensors. This low-cost and high-performance sensor can be adopted by any system wishing to detect the temperature. 


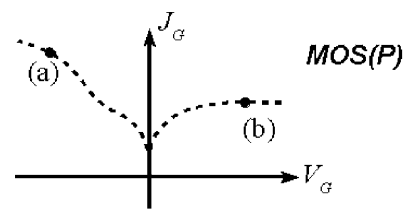

(a) At strong accumulation

(b) At deep depletion
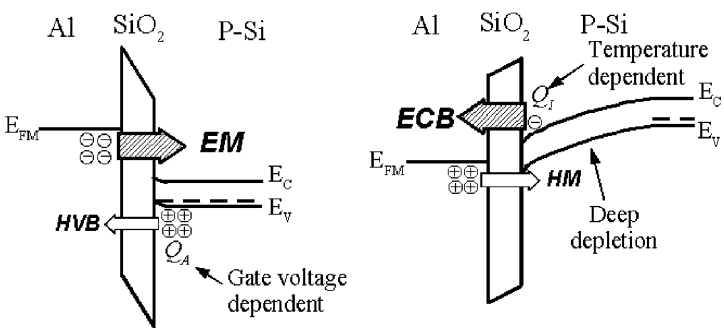

Fig. 1. Schematic band diagrams for an MOS tunneling diode biased (a) at accumulation and (b) at inversion.

\section{PRINCIPLE AND EXPERIMENTS}

The principles behind an MOS tunneling temperature sensor are simple ones. The properties of an MOS capacitor fabricated on a low-doped p-type substrate has a strongly oxide thickness-dependent. In the accumulation region, if $t_{\mathrm{ox}}$ exceeds $4 \mathrm{~nm}$, charge transport is dominated by Fowler-Nordheim $(\mathrm{F}-\mathrm{N})$ tunneling which is temperature-independent. For a thin oxide (for example, $t_{\mathrm{ox}}=2 \mathrm{~nm}$ ), direct tunneling dominates the gate current transportation at the low voltage regime [11]. However, at the inversion region, the gate current is not only limited by the oxide thickness but is also by the minority carrier generation. The inversion gate current saturates if the minority carrier generation rate is below the tunneling rate. Since minority carriers are temperature-sensitive, the saturation inversion gate current $\left(J_{\mathrm{G}_{-} \mathrm{SAT}}\right)$ changes with the substrate temperature. This characteristic is applied to on-chip temperature detection.

Fig. 1 illustrates the current mechanisms of an MOS (P) tunneling diode biased at both accumulation and inversion. Similar to a forward-biased p-n diode, gate current at accumulation is dominated by electron direct tunneling under the low field or by $\mathrm{F}-\mathrm{N}$ tunneling under the high field from the metal gate (EM) [12]. Since neither the hole concentration on the silicon surface and the gate electron concentration are substrate temperature ( $T_{\text {sub }}$ )-dependent, $J_{G}$ is only dependent on $V_{G}$. At inversion, $J_{G}$ is controlled by the total amount of electrons directly tunneling from the silicon conduction band (ECB) [13]. Raising $T_{\text {sub }}$ increases the electron concentration on the silicon surface conduction band, and $J_{\mathrm{G}_{-} \mathrm{SAT}}$ increases with $T_{\text {sub }}$.

Experimental results indicated no inversion oxide breakdown since low-doped, 1-5 $\Omega \cdot \mathrm{cm}$, and p-type silicon wafers were used. Triggering inversion oxide breakdown on such kind of MOS diode is hard, and does occur until silicon breakdown [14]. The silicon breakdown voltage $\left(V_{\mathrm{BD}}\right)$ is defined by

$$
V_{\mathrm{BD}}=\frac{\varepsilon_{\mathrm{Si}}}{2 q N_{A}} E_{\mathrm{BD}}^{2}
$$

where $\varepsilon_{\mathrm{Si}}, q, N_{A}$, and $E_{\mathrm{BD}}$ denote silicon permittivity, electron charge, doping density, and silicon breakdown field $(0.3$ $\mathrm{MV} / \mathrm{cm})$, respectively [15]. For the samples with $V_{G}$ below 15
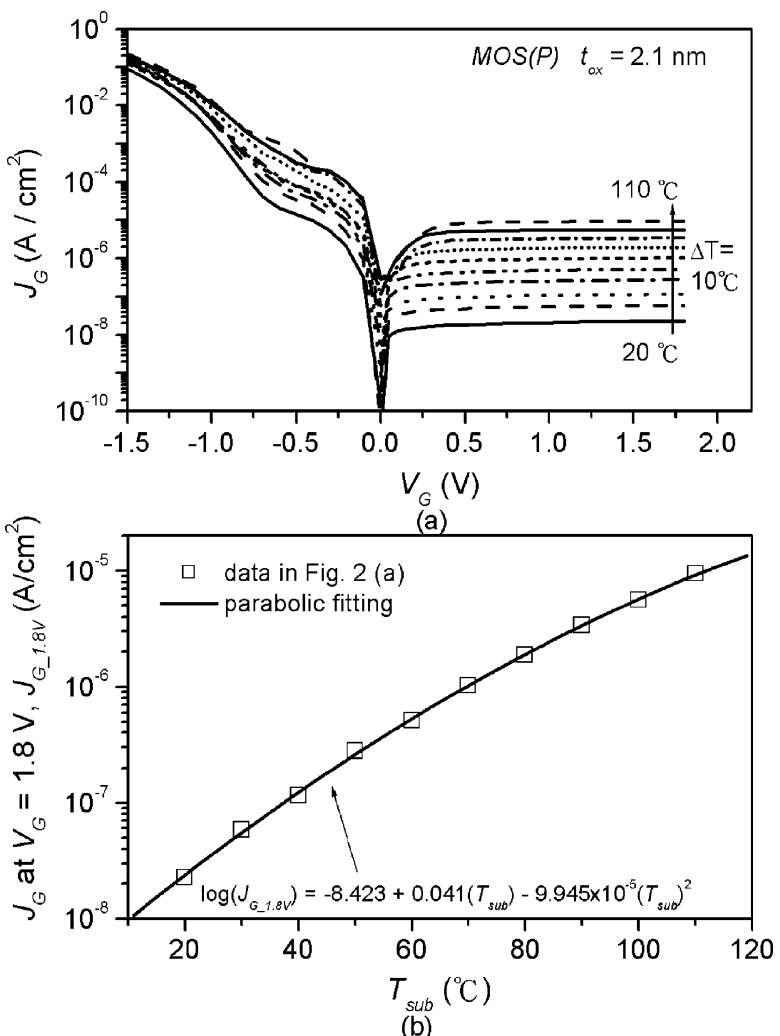

Fig. 2. (a) $J_{G}-V_{G}$ curves of a $150 \times 150 \mu \mathrm{m}^{2}$ MOS tunneling temperature sensor measured at various temperatures. While the gate voltage is positive, the current delimited by minority carrier (electron) generation rate is changed with $T_{\text {sub. }}$ (b) $J_{1.8 V}-T_{\text {sub }}$ data of the sensor in (a). Empirical parabolic curve fits the data points well.

$\mathrm{V}$, the maximum E-field on the silicon surface is far below the silicon breakdown field. Since the gate current characteristics of MOS capacitors $\left(t_{\mathrm{ox}} \approx 2 \mathrm{~nm}\right.$ ) resemble those of $\mathrm{p}$-n diodes, they are sometimes called MOS tunneling diodes.

For all experiment samples, the oxides were first grown in a rapid thermal processor and then nitrogen annealed. Oxide thickness was determined using an ellipsometer with a fixed refractive index of $n=1.458$. Following aluminum evaporation, devices with $150 \times 150 \mu \mathrm{m}^{2}$ and $2500 \times 2500 \mu \mathrm{m}^{2}$ gate areas were patterned via photolithography and wet etching. The large area devices are required for the possible direct application of a constant current to an MOS sensor in this paper. When the sensors are integrated into circuits, the area is very small. Following by back oxide stripping, aluminum was evaporated again on the back of the wafer. Some devices were post-metallization annealed to reduce the effect of the interface state on the current.

Temperature responses of the MOS tunneling diodes are described using current density versus voltage $\left(J_{G}-V_{G}\right)$ curves, current density versus time $\left(J_{G}-t\right)$ curves and voltage versus time $\left(V_{G}-t\right)$ curves by measuring samples on a hot chuck. The chuck temperature treated as the substrate temperature ranges from $20{ }^{\circ} \mathrm{C}$ to $110^{\circ} \mathrm{C}$. As a certain constant current is applied to the sensor, the gate voltage $\left(V_{G}\right)$ must change rapidly with the substrate temperature to maintain the same current quantity. This paper applies both of the constant-voltage and constant-current characteristics to on-chip temperature detection. 


\section{RESULTS AND DisCUSSIONS}

\section{A. Temperature Responses of MOS Tunneling Sensors Under Constant-Voltage Operation}

Fig. 2(a) displays typical $J_{G}-V_{G}$ curves of an MOS tunneling temperature sensor $\left(t_{\mathrm{ox}}=2.1 \mathrm{~nm}\right)$ at different substrate temperatures. When such a sensor is biased at accumulation, $J_{G}$ is $V_{G}$ dependent. On the other hand, $J_{G}$ changes significantly with $T_{\text {sub }}$ at inversion. A $10{ }^{\circ} \mathrm{C}$ increase in $T_{\text {sub }}$ almost doubles $J_{\mathrm{G} \_\mathrm{SAT}}$, and $J_{\mathrm{G} \_\mathrm{SAT}}$ increases more than $500 \mathrm{X}$ when $T_{\mathrm{sub}}$ is raised from $20{ }^{\circ} \mathrm{C}$ to $110{ }^{\circ} \mathrm{C}$. We take $J_{G}$ at $V_{G}=1.8 \mathrm{~V}$ $\left(J_{G_{-} 1.8 \mathrm{~V}}\right)$ as a temperature indicator. $J_{G_{-1} .8 \mathrm{~V}}-T_{\text {sub }}$ relationship is manifested in Fig. 2(b). An empirical parabolic function, i.e., that is

$$
\log \left(J_{G_{-} 1.8 V}\right)=-8.423+0.041\left(T_{\text {sub }}\right)-9.945 \times 10^{-5}\left(T_{\text {sub }}\right)^{2}
$$

fits the $J_{G_{-} 1.8 \mathrm{~V}}$ data well, and $T_{\text {sub }}$ can be easily obtained by (3), shown at the bottom of the page. Notably, the value of constant in (2) can vary from device to device among devices. This paper changed $T_{\text {sub }}$ regularly and continuously measured the $J_{G_{-} 1.8 \mathrm{~V}}$ of one MOS temperature sensor. The sensor was heated and cooled twice. Each cycle including seven states lasts for $5000 \mathrm{~s}$. Each of the states was separated by $10{ }^{\circ} \mathrm{C}$. The $J_{G_{-} 1.8 V^{-}} t$ curve is illustrated in Fig. 3(a). Since the heater used here is a proportional-integral- derivative-controlled, gate current damping occurs in each state, particularly in the initial duration of each state. However, once $T_{\text {sub }}$ is stable, $J_{G_{-1} 1.8 \mathrm{~V}}$ remains constant. Between states, $J_{G_{-} 1.8 \mathrm{~V}}$ increases with increasing $T_{\text {sub }}$. Notably, the values of $J_{G_{-} 1.8 \mathrm{~V}}$ in the first and the second cycles are almost identical at the same $T_{\text {sub. An }}$ MOS temperature sensor is reliable even though it suffers stress at high temperatures. Fig. 3(b) illustrates stable $J_{G_{-1} .8 \mathrm{~V}}$ data at each stage and a fitted parabolic curve. A curve identical to that in Fig. 2(b) is also shown in Fig. 3(b) to provide a comparison. These two curves have the same coefficients except for the constant term. The discrepancy involving the constant term implies that the gate current of an MOS tunneling temperature sensor could differ among samples, but that their temperature responses are similar. The discrepancy results from the variations in oxide thickness, defect density, and interface traps. This finding indicates that composing numerous MOS temperature sensors into a temperature sensor array was very difficult if the differences among sensors could not be eliminated. In a later section i.e., namely, Section III-C, a method of indirect temperature converting via gate current gains $\left(A_{G}\right)$ is demonstrated that solves this problem.

\section{B. Temperature Responses of MOS Tunneling Sensors Under Constant-Current Operation}

Fig. 4 illustrates the $I_{G}-V_{G}$ characteristics of an MOS temperature sensor $\left(t_{\mathrm{ox}}=2.1 \mathrm{~nm}\right)$ with an area of $2500 \times 2500$

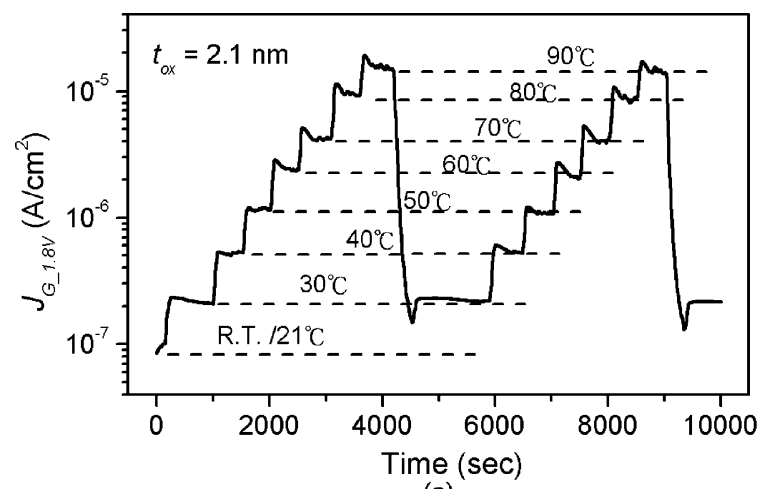

(a)

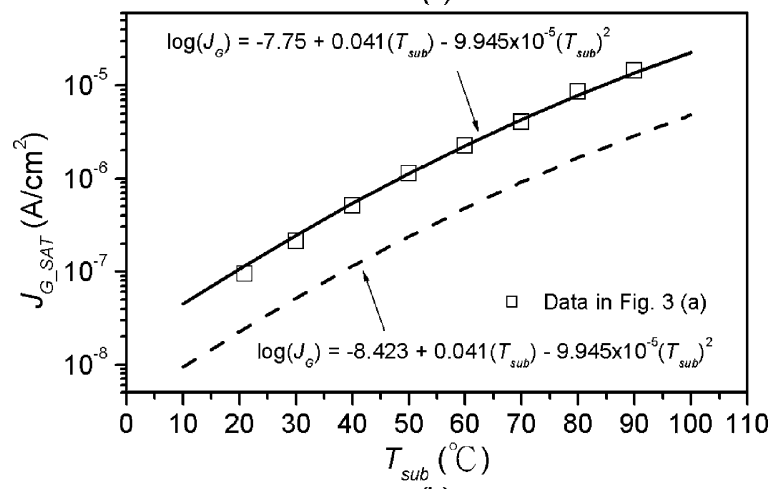

(b)

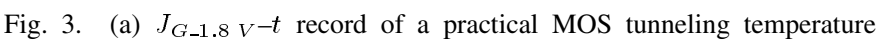
sensor biased under constant voltage. The record contains two heating-up and cooling-down cycles. In each cycle, there are seven stages separated by $10^{\circ} \mathrm{C}$. (b) Stable $J_{G-1.8 \mathrm{~V}}$ data from each stage follow the same temperature response curve as shown in Fig. 2(b), except the constant term.

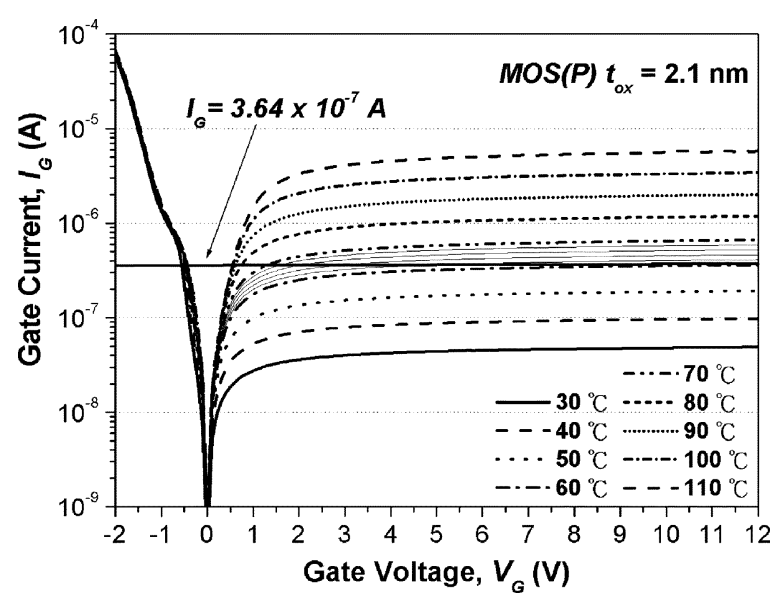

Fig. 4. $I_{G}-V_{G}$ curves of a $2500 \times 2500 \mu \mathrm{m}^{2}$ MOS tunneling temperature sensor measured at various temperatures. A constant current of $3.64 \times 10^{-7} \mathrm{~A}$ is plotted for demonstrating the possible voltage detection under various temperatures.

$\mu \mathrm{m}^{2}$ measured at different substrate temperatures. As a certain constant current is applied to the sensor (solid line in Fig. 4), the

$$
T_{\text {sub }}=\frac{0.041-\sqrt{(0.041)^{2}-4 \times\left(9.945 \times 10^{-5}\right) \times\left(8.423+\log \left(J_{G_{-} 1.8 V}\right)\right)}}{2 \times\left(9.945 \times 10^{-5}\right)}\left({ }^{\circ} \mathrm{C}\right) .
$$




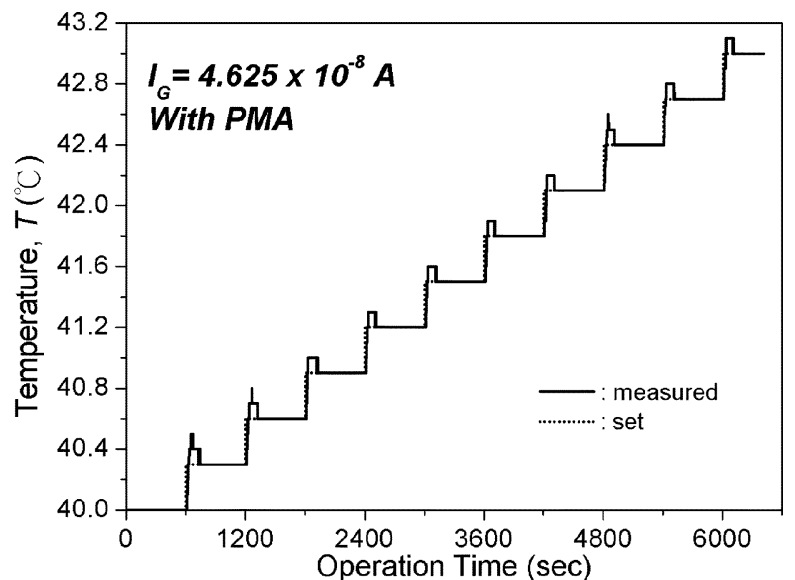

(a)

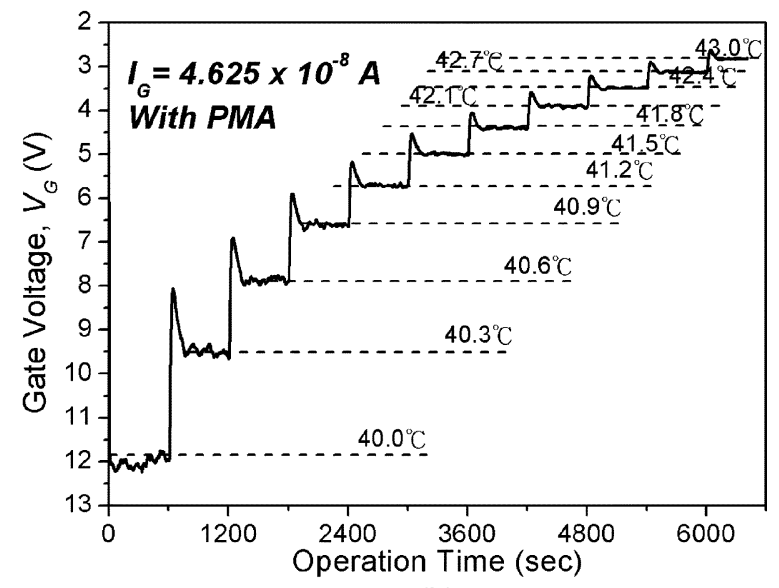

(b)

Fig. 5. (a) Variations of temperature ranging from a $40 \sim 43{ }^{\circ} \mathrm{C}$ through $0.3^{\circ} \mathrm{C} /$ step increment setting. The dotted line is the temperature programmed by controller and the solid line is real temperature measured in chuck. (b) Gate voltage $\left(V_{G}\right)$ varies step by step with time when a $0.3{ }^{\circ} \mathrm{C}$ step is applied to the sensor under constant current bias operation.

sustained $V_{G}$ across the device must change rapidly with substrate temperature to maintain the same current. Under such a constant current bias, flatter current-voltage $(I-V)$ curves owing to temperature variation in the inversion region have larger gate voltage changes. The sensors under the constant-current operation could always achieve a temperature sensitivity exceeding $-2 \mathrm{~V} /{ }^{\circ} \mathrm{C}$.

Fig. 5 shows a schematic assessment of a practical MOS tunneling temperature sensor. The figure illustrates the $V_{G}$ versus time for this temperature sensor under constant-current operation. The bias current magnitude of this sensor is around 46.25 $\mathrm{nA}$. The chuck temperature was treated as the same temperature of substrate at ranges from $40^{\circ} \mathrm{C}$ to $43^{\circ} \mathrm{C}$. The dotted line in Fig. 5(a) illustrates the temperature programmed by the controller, and the solid line denotes the real temperature measured in the chuck. The sensor was heated from $40{ }^{\circ} \mathrm{C}$ to $43{ }^{\circ} \mathrm{C}$ and stabilized using a $0.3^{\circ} \mathrm{C}$ gap. Fig. 5(b) reveals that the $V_{G}$ varies in a stepwise fashion with time when a $0.3{ }^{\circ} \mathrm{C}$ step is applied to the sensor. The continuous gate voltage clearly determines the substrate temperature. A $1^{\circ} \mathrm{C}$ increment (from $40{ }^{\circ} \mathrm{C}$ to $41^{\circ} \mathrm{C}$ ) reduced the $V_{G}$ by around $5.5 \mathrm{~V}$ (from 11.8 to $6.3 \mathrm{~V}$ ); that is,

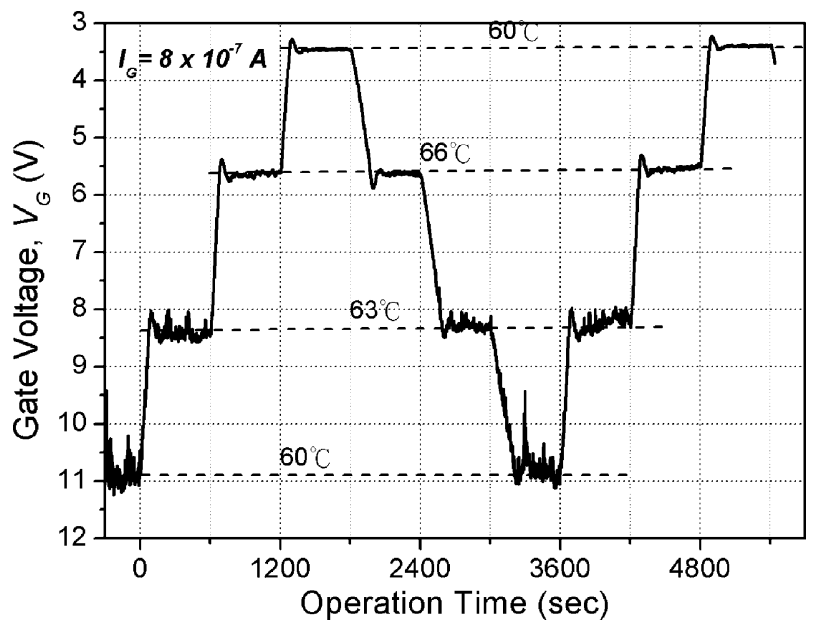

Fig. 6. Voltage levels in the heating-up and cooling-down cycles of an MOS tunneling temperature sensor under constant current bias operation. The gate voltage levels are identical at the same chuck temperature.

the temperature sensitivity is around $-5.5 \mathrm{~V} /{ }^{\circ} \mathrm{C}$. The temperature sensitivity decreases with increasing substrate temperature. With limiting the detection range $\left(40^{\circ} \mathrm{C} \sim 41.5^{\circ} \mathrm{C}\right)$, this sensor could achieve temperature sensitivity exceeding $-2 \mathrm{~V} /{ }^{\circ} \mathrm{C}$. A high sensitive MOS tunneling temperature sensor always faces the problem of low detecting range because the voltage drops rapidly. This problem can be overcome by changing the constant-current biasing level to enlarge the detection voltage range.

Fig. 6 illustrates the voltage levels in the heating and cooling cycles. The temperature sensor was heated from $60{ }^{\circ} \mathrm{C}$ to $69^{\circ} \mathrm{C}$ and stabilized using a $3{ }^{\circ} \mathrm{C}$ gap. The sensor then was cooled from $69^{\circ} \mathrm{C}$ to $60{ }^{\circ} \mathrm{C}$ and stabilized using the same gap. The controller, heating, and cooling cycles then were repeated. The gate voltage levels are identical at the same chuck temperature. A one-to-one relation exists between the output gate voltage and the substrate temperature. The high sensitivity MOS tunneling temperature sensor thus has good uniformity. From the previous discussion, while $V_{G}$ is smaller than $15 \mathrm{~V}$, the maximum E-field on the silicon surface is far below the silicon breakdown field $(0.3 \mathrm{MV} / \mathrm{cm})$. The entire operating voltage range in the present experiments is below $12 \mathrm{~V}$, and thus, the operation of the proposed temperature sensor is unaffected by such gate voltage. This figure shows that uniformity of an MOS temperature sensor could be maintained despite being subjected to high voltage stress (up to $11 \mathrm{~V}$ ). Moreover, the $I-V$ curve of this temperature sensor is identical to before a high voltage stress. Consequently, although the device was high voltage stressed, it is believed to be reliable.

\section{Indirect Temperature Conversion Method for a Temperature Sensor Array}

A big challenge in creating a temperature sensor array based on numerous temperature sensors is that saturation current may differ among sensors. Oxide thickness variation, defect densities, and interface traps all may contribute to gate current discrepancies. This paper proposes a new method of using current gain to solve this problem. Using current gain as the detecting parameter can reduce the variations in current levels owing to oxide thickness nonuniformity, and different gate area. 


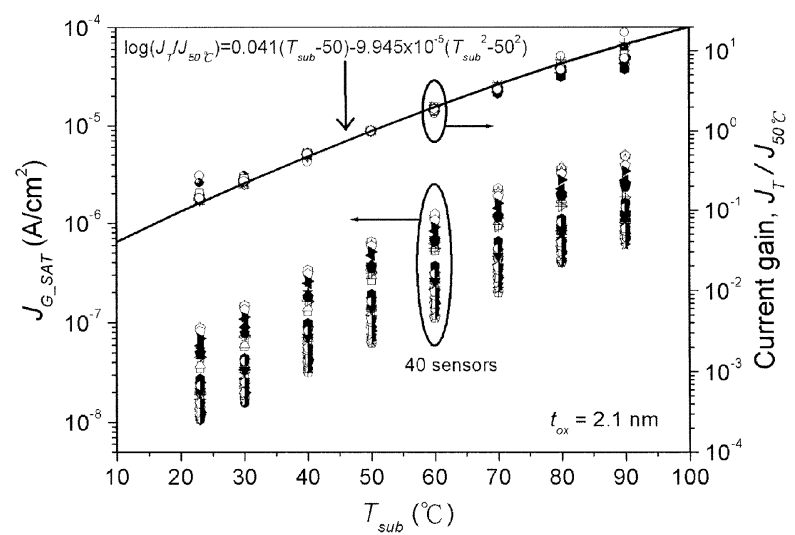

Fig. 7. Temperature responses of 40 MOS temperature sensors under constant voltage bias operation represented by gate current density and by current gain. The huge discrepancies among the sensors can be reduced by current gain method.

Fig. 7 shows the $J_{G_{-1} .8 \mathrm{~V}}$ distribution of 40 MOS temperature sensors $\left(t_{\mathrm{ox}}=2.1 \mathrm{~nm}\right)$ in an array. $J_{G_{-} 1.8 \mathrm{~V}}$ varies over an order. That is, if only a $J_{G}-T_{\text {sub }}$ converter handles currents coming from the 40 sensors, the current variation will demonstrate an unacceptable error of $40^{\circ} \mathrm{C}$. The same figure demonstrates gate current gains $\left(A_{G}\right)$ of the sensors. $J_{G_{-} 1.8 \mathrm{~V}}$ at $50^{\circ} \mathrm{C}$ serves as the reference. Moreover, the $A_{G}$ distribution in the logarithm scale is described using a parabolic fitting curve. If $A_{G}$ distribution is used for $T_{\text {sub }}$ detection, the error can be significantly suppressed from $40{ }^{\circ} \mathrm{C}$ to $5{ }^{\circ} \mathrm{C}$. At high temperature ( $T_{\text {sub }}>80^{\circ} \mathrm{C}$ ), a temperature difference is expected between the silicon surface and the hot chuck for the stretch-out data.

The unique fitting curve in Fig. 7 indicates an indirect temperature conversion method for a sensor array. Before measuring $T_{\text {sub }}$, each sensor should be individually calibrated at a specific temperature $\left(T_{\text {spec }}\right)$, and its gate current $\left(J_{\text {spec }}\right)$ is then recorded for future temperature calculation. Equation (2) displays the expression of $A_{G}$ as follows:

$$
\begin{aligned}
\log \left(\frac{J_{T}}{J_{\text {spec }}}\right)=\log \left(A_{G}\right) & =0.041\left(T_{\text {sub }}-T_{\text {spec }}\right) \\
- & 9.945 \times 10^{-5}\left(T_{\text {sub }}^{2}-T_{\text {spec }}^{2}\right) .
\end{aligned}
$$

Notably, $J_{T}$ denotes the gate current density when the substrate temperature is $T_{\text {sub }}$. Then $T_{\text {sub }}$ is obtainable by solving (4). Fabrication processes may slightly alter the coefficients in (2), but can be experimentally determined. Equation (4) gives a general solution for such kinds of an MOS temperature sensor array, and the solution is much easier for an array to embody.

\section{Model for MOS Devices Biased at Inversion Region}

The model used to fit the gate current can be expressed as

$$
\log J_{T}=a_{0}+a_{1} T_{\mathrm{sub}}+a_{2} T_{\mathrm{sub}}^{2}+b_{1} V_{G}+b_{2} V_{G}^{2}
$$

where the values $a_{0}, a_{1}, a_{2}, b_{1}$, and $b_{2}$ are constants. Fig. 8 shows $I-V$ curves fitted by second-order polynomials. The dotted lines denote the measured gate currents under different substrate temperatures while the solid lines are the fitted gate currents based on (5). Within the sensor operating range

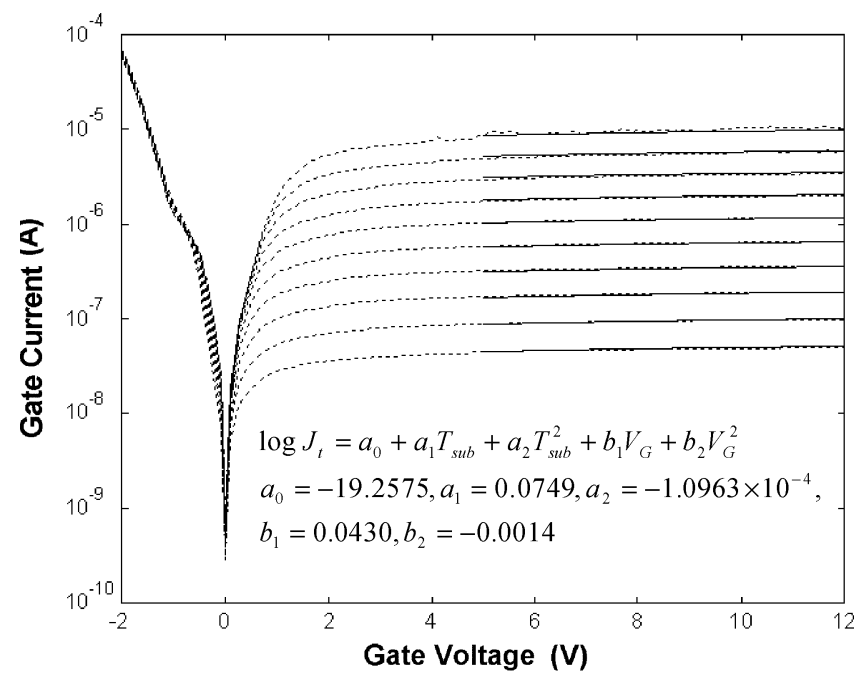

Fig. 8. $I-V$ curves fitted by second-order polynomials.

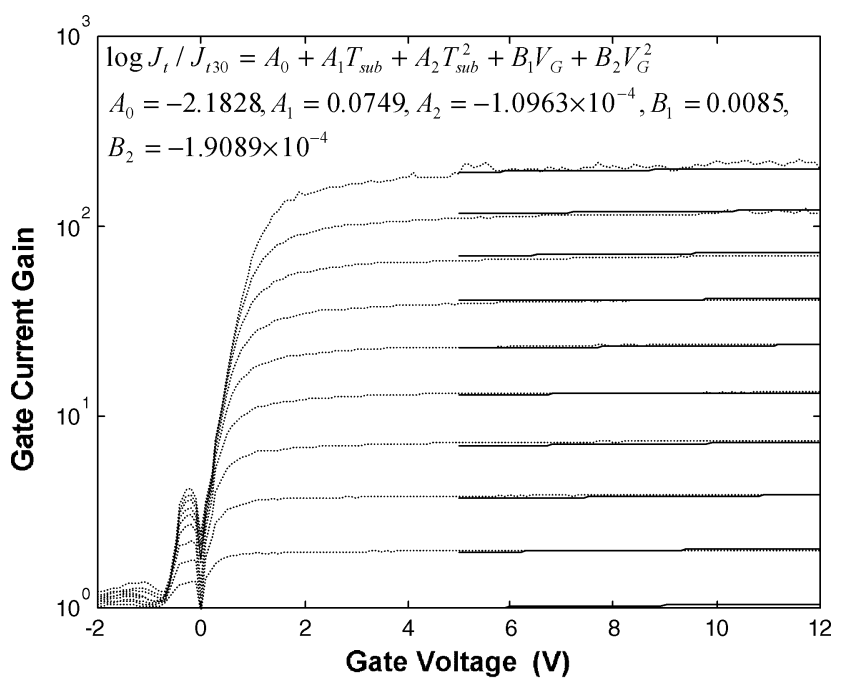

Fig. 9. Gate current gain versus the gate voltage curves that will be fitted by second-order polynomials.

$(+5 \mathrm{~V} \sim+12 \mathrm{~V})$, the two curves are almost overlapping. That is, the proposed model provides a perfect fit. From previous discussion, the gate current gain, rather than the absolute gate current, was used for assessing the temperature. The gate current gain algorithm could overcome the process variation. The gate current gain fitted using second-order polynomials can be expressed as

$$
\log \frac{J_{T}}{J_{T, 30}}=A_{0}+A_{1} T_{\text {sub }}+A_{2} T_{\text {sub }}^{2}+B_{1} V_{G}+B_{2} V_{G}^{2}
$$

where the values $A_{0}, A_{1}, A_{2}, B_{1}$, and $B_{2}$ are constants and $J_{T, 30}$ is the gate current when the substrate temperature is $30{ }^{\circ} \mathrm{C}$. Fig. 9 illustrates the gate current gain versus the gate voltage curves fitted by second-order polynomials. The proposed model is quite simple. Fig. 9 shows the measured and calculated curves, which reveal a perfect fit. Since different sensors share the same coefficients in this model, the current gain algorithm is believed to be useful for temperature detection. 


\section{E. Analysis of Temperature Sensors' Sensitivity}

Differentiating the equation as proposed above can obtain the following:

$\frac{J_{T, 30}}{J_{T}} \cdot \frac{1}{J_{T, 30}} \cdot \frac{\partial J_{T}}{\partial T_{\mathrm{sub}}}=A_{1}+2 A_{2} T_{\mathrm{sub}}+B_{1} \frac{\partial V_{G}}{\partial T_{\mathrm{sub}}}+2 B_{2} \frac{\partial V_{G}}{\partial T_{\mathrm{sub}}}$.

Rearranging (7) leads to

$$
\partial T_{\text {sub }}=\frac{1}{A_{1}+2 A_{2} T_{\text {sub }}} \cdot \frac{\partial J_{T}}{J_{T}}-\frac{B_{1}+2 B_{2} V_{G}}{A_{1}+2 A_{2} T_{\text {sub }}} \cdot \partial V_{G} .
$$

Lower substrate temperature $\left(T_{\mathrm{sub}}\right)$ causes lower gate current $\left(J_{T}\right)$. The above equation reveals that larger substrate temperature deviation $\left(\partial T_{\mathrm{sub}}\right)$ will be produced by the first term, which implies that the temperature sensors have a lower sensitivity under low temperature situations. Additionally, $\partial J_{T}$ is determined based on the detecting circuit's resolution. Higher resolution produces lower substrate temperature deviation, and improves the sensitivity of the temperature sensor. The second term derives from the usage of the voltage information of the gate. The $\partial V_{G}$ term is also determined based on the detecting circuit resolution. The detecting circuit forces the second term to eliminate the first term by producing a suitable $V_{G}$, resulting in a smaller substrate temperature deviation and improvement in the sensitivity of the temperature sensor. Correspondingly, a higher resolution produces better eliminating effect, thus a higher temperature sensitivity can be obtained.

Fig. 10(a) and (b) illustrates the gate voltage and the variation of temperature sensitivity with the substrate temperature, respectively. The sensitivities were determined by the semi-experiment approach based on the $I-V$ curve data measured under different temperatures. Both the gate voltage and the temperature sensitivity decrease with increasing substrate temperature in region 1 (from $36.9^{\circ} \mathrm{C}$ to $37.4^{\circ} \mathrm{C}$ ). Moreover, the gate voltage reaches the lower limit $(5 \mathrm{~V})$, as the temperature equals $37.4^{\circ} \mathrm{C}$. The bias level of the constant current source must change to prevent the sensor from operating out of the detection range ( $5 \sim 12 \mathrm{~V}$ ). Different regions have different bias current levels. Moreover, this change in bias current always keeps the temperature sensitivity above $10 \mathrm{~V}^{\circ} \mathrm{C}$ in our paper theoretically.

On the other hand, the experimental approach is also used to realize the temperature sensors except the above semi-experimental way. Fig. 11 shows that the measured gate voltages varied with the substrate temperature for one temperature sensor. Moreover, the resolution of the sensor under the constant current operation is at least $-2 \mathrm{~V} /{ }^{\circ} \mathrm{C}$. However, the resolution reduces with decreasing gate voltage, and making the noise consideration] more important. To avoid this noise effect, the sensors should be biased to operate under an appropriate voltage range. For example, the current level was changed from $1.69 \times 10^{-6} \mathrm{~A} / \mathrm{cm}^{2}$ to $1.82 \times 10^{-6} \mathrm{~A} / \mathrm{cm}^{2}$, as illustrated in Fig. 11 to maintain the voltage range within $19 \sim 13$ volts when the temperature changes from $35{ }^{\circ} \mathrm{C}$ to $37{ }^{\circ} \mathrm{C}$. Bias current levels differ among regions. This device is clearly observed to have high sensitivity.

\section{F. Temperature Distribution Detection}

This study demonstrated the real-time temperature distribution for on-chip detection. Multi-points on the wafer are mea-

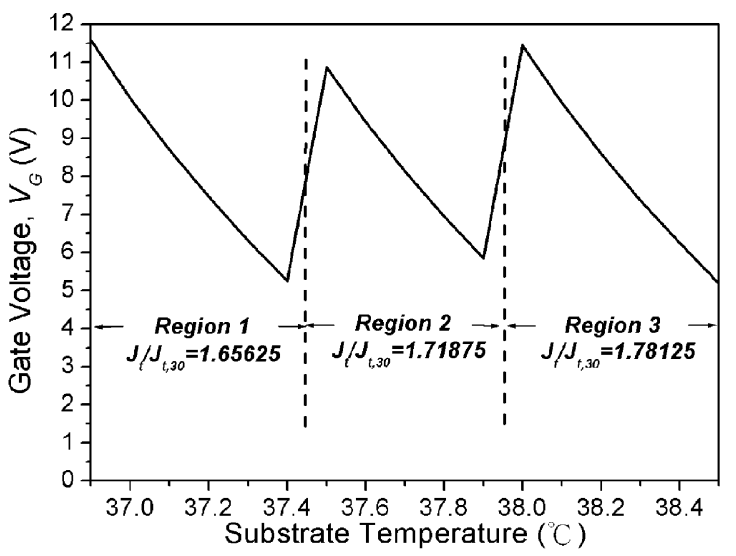

(a)

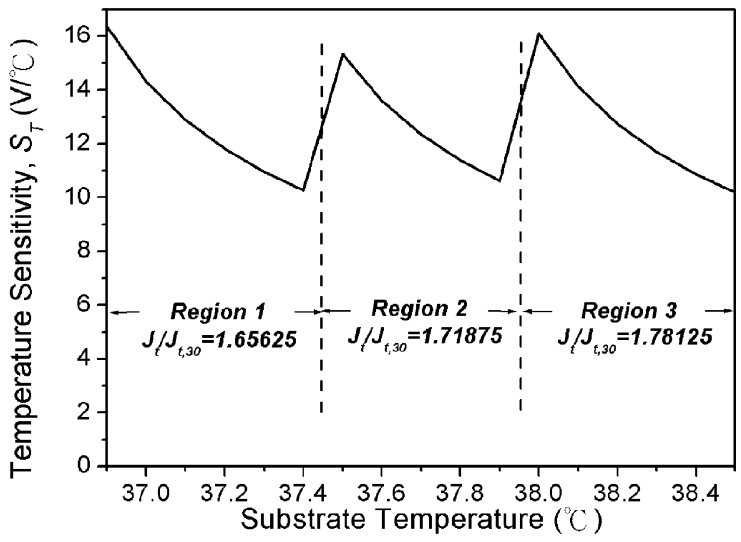

(b)

Fig. 10. (a) Variations of the gate voltage and (b) the temperature sensitivity as a function of the substrate temperature according to the fitted polynomials under three different constant current biases operation.

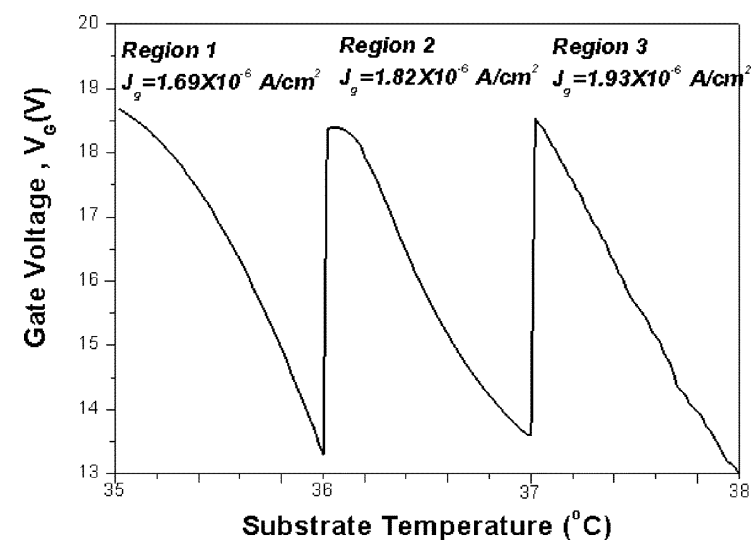

Fig. 11. Experimental observation of the variations of gate voltage as a function of the substrate temperature for an MOS sensor measured at three different constant current biases operation.

sured, and the current gain method is used. The temperature distribution on the wafer can also be pictured. The currents of three dies are measured at $30{ }^{\circ} \mathrm{C}$, and serve as current references. Fig. 12(a) illustrates the current densities of the three points versus time. The currents rapidly rise when the heat source approaches the chip. The current discrepancies can be taken away using the current gain method, as shown in Fig. 12(b). The ratio of $J_{T} / J_{T, 30}$ can indicate the temperature situation. When the currents of multi-points are measured already, the temperature 


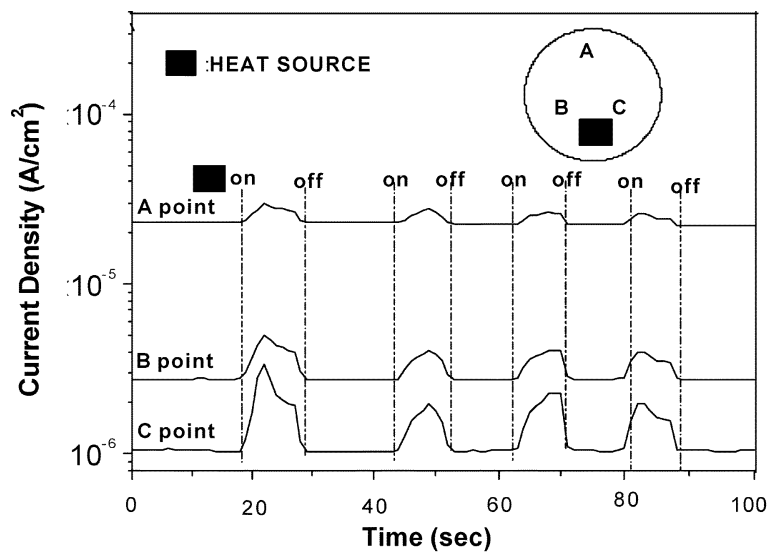

(a)

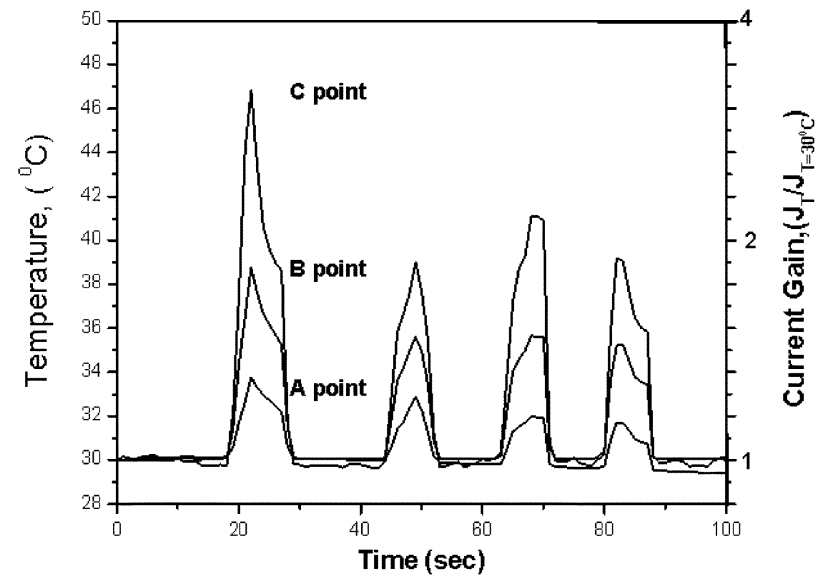

(b)

Fig. 12. Simultaneous responses of (a) the current densities and (b) the current gains and the corresponding interpreted real time temperature for three temperature sensors on a wafer.

distribution on the chip will be decided. Notably, under the bias of $2 \mathrm{~V}$, the $A_{2} T_{\text {sub }}^{2}, B_{1} V_{G}$, and $B_{2} V_{G}^{2}$ terms in (6) can be ignored. Consequently, the model used here to fit the temperature distribution can be simplified as follows:

$$
T_{\text {sub }} \cong \frac{1}{A}\left(\log \frac{J_{T}}{J_{T, 30}}-B\right)
$$

where $A$ and $B$ are constants. Fig. 12(b) also shows the interpreted temperature distribution on the wafer versus the real time. With further improvement in the stability and reliability, the integration of temperature sensors on a chip is expected to detect the entire real time temperature distribution on the wafer. temperature sensitivity exceeding $-2 \mathrm{~V} /{ }^{\circ} \mathrm{C}$. Such sensitivity is one thousand times larger than that of conventional systems. As for a temperature sensor array, this paper proposes a method using gate current gain, rather than absolute gate current, to obtain temperature. The proposed method eliminates gate current discrepancy among sensors. This paper also demonstrates the possible application of detecting the multi-points temperatures on a wafer. The proposed low-cost and high-performance sensor is useful for on-chip temperature detection application.

\section{REFERENCES}

[1] C. N. Liao, C. Chen, and K. N. Tu, "Thermoelectric characterization of Si thin films in silicon-on-insulator wafer," J. Appl. Phys., vol. 86, no. 8, pp. 3204-3208, Sept. 1999.

[2] H. Sanchez, B. Kuttanna, T. Olson, M. Alexander, G. Georosa, R. Philip, and J. Alvarez, "Thermal management system for high performance power PC microprocessors," in Proc. Compcon, 1997, pp. 325-330.

[3] A. Syal, V. Lee, A. Ivanov, and J. Altet, "CMOS differential and absolute thermal sensors," in Proc. On-Line Testing Workshop, 2001, pp. 127-132.

[4] V. Szekely, C. Marta, Z. Kohari, and M. Rencz, "CMOS sensors for on-line thermal monitoring of VLSI circuits," IEEE Trans. VLSI Syst., vol. 5, pp. 270-276, Sept. 1997.

[5] J. Altet, A. Rubio, S. Dilhaire, E. Schaub, and W. Claeys, "BiCMOS thermal sensor circuit for built-in test purposes," Electron. Lett., vol. 34, pp. 1307-1309, June 1998.

[6] A. Bakker and J. H. Huijsing, "Micropower CMOS temperature sensor with digital output," IEEE Trans. Solid-State Circuits, vol. 31, pp. 933-937, July 1996.

[7] G. Fisher, J. C. Daly, C. W. Recksiek, and K. D. Friedland, "A programmable temperature monitoring device for tagging small fish: A prototype chip development," IEEE Trans. VLSI Syst., vol. 5, pp. 401-407, Dec. 1997.

[8] J. L. Merino, S. A. Bota, A. Herms, E. Cabruja, X. Jorda, A. Ferre, J. Bigorra, J. Samitier, M. Vellvehi, and J. Bausells, "Smart temperature sensor for on-line monitoring in automotive applications," in Proc. On-Line Testing Workshop, 2001, pp. 122-126.

[9] V. F. Mitin, V. V. Kholevchuk, R. V. Konakova, and N. S. Boltovet, "Temperature microsensors," in Proc. Semiconductor Conf., vol. 2, 1999, pp. 495-498.

[10] Y. H. Shih and J. G. Hwu, "An on-chip temperature sensor by utilizing a MOS tunneling diode," IEEE Electron Device Lett., vol. 22, pp. 299-301, June 2001.

[11] T. P. Chen, S. Li, S. Fung, and K. F. Lo, "Interface generation by FN injection under dynamic oxide field stress," IEEE Trans. Electron Devices, vol. 45, pp. 1920-1926, Sept. 1998.

[12] A. Yassine, H. E. Nariman, and K. Olasupo, "Field and temperature dependence of TDDB of ultrathin gate oxide," IEEE Electron Device Lett., vol. 20, pp. 390-392, Aug. 1999.

[13] W. C. Lee and C. Hu, "Modeling gate and substrate currents due to conduction- and valence-band electron and hole tunneling," in Symp. VLSI Tech. Dig., June 2000, pp. 198-199.

[14] C. G. Fonstad, Microelectronic Devices And Circuits. New York: McGraw-Hill, 1994, p. 153.

[15] S. M. Sze, VLSI Technology, 2nd ed. New York: McGraw-Hill, 1988, p. 656.

\section{CONCLUSION}

A high sensitivity temperature sensor that used an MOS tunneling diode structure was designed in this paper. It has the advantage of being CMOS technology compatible. Since the sensor is biased at constant-voltage, the gate current was increased over 500 times when the sensor was heated from 20 ${ }^{\circ} \mathrm{C}$ to $110^{\circ} \mathrm{C}$. Additionally, since the sensor operates in a constant-current situation and with limiting output voltage range, the proposed MOS tunneling temperature sensor could achieve

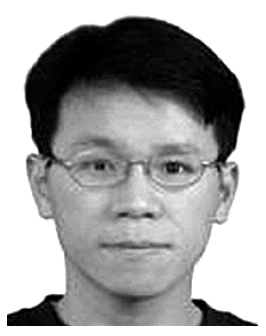

Yen-Hao Shih was born in Changhua City, Taiwan, R.O.C., in 1974. He received the B.S. and the Ph.D. degrees in electron engineering from National Taiwan University, Taipei, in 1997 and 2002, respectively.

In 2002, he joined Emerging Central Lab, Macronix International Company, Ltd., Hsinchu, Taiwan. His research fields are ultrathin gate oxide, nitride thin films, reliability, and nitride storage flash memories. 


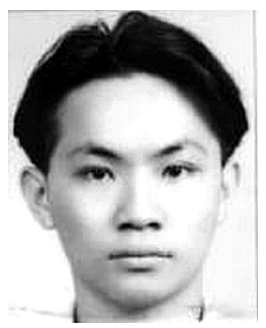

Shian-Ru Lin was born in Nantou, Taiwan, R.O.C., in 1978. He received the B.S. degree in electronic engineering from National Taiwan University (NTU) of Science and Technology, Taipei, in 2000, and the M.S. degree in electronic engineering from NTU, in 2003.

He joined Realtek Semiconductor Corporation, Hsinchu, Taiwan, in 2003. His research interests include the MOS device, on-chip temperature sensors, and high-speed ADCs.

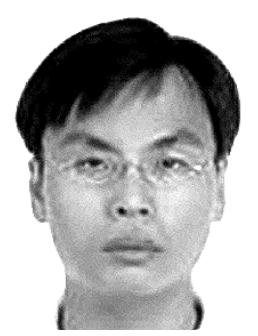

Tsung-Miau Wang was born in Changhua, Taiwan, R.O.C., in 1980. He received the B.S. degree in electronic engineering from National Chiao-Tung University, Hsinchu, Taiwan, in 2002. After his graduation, he pursued the M.S. degree in electronic engineering in National Taiwan University, Taipei, for one year and he was then qualified to enter the Ph.D. program in 2003.

His research major is MOS devices, especially on-chip temperature sensors.

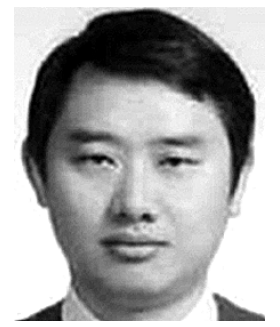

Jenn-Gwo Hwu (SM'99) was born in Taiwan, R.O.C., on August 29, 1955. He received the B.S. degree in electronic engineering from National Chiao-Tung University, Hsinchu, Taiwan, in 1977 and the M.S. and Ph.D. degrees in electrical engineering from National Taiwan University (NTU), Taipei, in 1979 and 1985, respectively.

He joined the faculty of NTU in 1981, where he is presently a Professor with the Department of Electrical Engineering and with the Graduate Institute of Electronics Engineering. From 1997 to 1998, he was the vice chairman of the Department of Electrical Engineering. From February 1, 2004, he was invited to be the Dean of the College of Electrical Engineering and Computer Science, National United University, Miaoli, Taiwan. His research is mainly on the preparation of reliable ultrathin gate oxide and the related MOS devices. He has experience in teaching the courses of circuits, electronics, solid-state electronics, semiconductor engineering, MOS capacitor devices, radiation effects on MOS systems, and special topics on oxide reliability.

Dr. Hwu was a recipient of the 1986, 1998, 1999, 2000, and 2002 Excellent Teaching Award sponsored by NTU. From 1988 to 1991, and 1993, he was a recipient of the Excellent Teaching Award sponsored by the College of Engineering, NTU. In 1991, he was also a recipient of the Outstanding Teaching Award sponsored by the Ministry of Education, R.O.C., and in 1987 and 2003 by the NTU. In 1999, he was the recipient of Jan Ten-You Paper Award by The Chinese Institute of Engineering, R.O.C. 